\title{
Depth Magnetization Profile of a Perpendicular Exchange Coupled System by Soft-X-Ray Resonant Magnetic Reflectivity
}

\author{
J. M. Tonnerre, ${ }^{1}$ M. De Santis, ${ }^{1, *}$ S. Grenier, ${ }^{1}$ H. C. N. Tolentino, ${ }^{1}$ V. Langlais, ${ }^{1,2}$ E. Bontempi,${ }^{3}$ \\ M. García-Fernández, ${ }^{4}$ and U. Staub ${ }^{4}$ \\ ${ }^{1}$ Institut Néel, CNRS \& Université Joseph Fourier, BP 166, F-38042 Grenoble Cedex 9, France \\ ${ }^{2}$ Departament de Fisica, Universitat Autónoma de Barcelona, Spain \\ ${ }^{3}$ Laboratorio di Chimica per le Tecnologie, Università di Brescia, via Branze, 25123 Brescia, Italy \\ ${ }^{4}$ Swiss Light Source, Paul Sherrer Institut, 5232 Villigen, Switzerland
}

(Received 30 July 2007; published 16 April 2008)

\begin{abstract}
The magnetic profile across the interface of a perpendicular exchange coupled $[\mathrm{NiO} / \mathrm{CoO}]_{3} / \mathrm{Pt}-\mathrm{Co} /$ $\mathrm{Pt}$ (111) system is investigated. The magneto-optic Kerr effect reveals a strong coupling between the antiferromagnetic (AFM) oxide and the ferromagnetic (FM) Pt-Co layer, by an increasing coercivity and a rotation of the easy magnetization axis of the FM layer along the AFM spins. Soft x-ray resonant magnetic reflectivity is used to probe the spatial distribution of the out-of-plane magnetization inside the oxide above its ordering temperature. It extends over $1 \mathrm{~nm}$ and exhibits a change of sign.
\end{abstract}

PACS numbers: 75.25.+z, 61.05.cm, 75.30.Gw, 75.70.Cn

The complex magnetic coupling at the interface of ultrathin films with different magnetic orders may give rise to unusual properties. The essential behavior depends critically on the modifications of the interfacial electronic and magnetic properties. Key issues concern the extension of the proximity effects on each side of the interface [1]. This is particularly crucial in exchange coupled ferromagneticantiferromagnetic (FM-AFM) systems, which exhibit exchange bias (EB), an important property in spin valves and magnetic tunnel junctions related devices. EB manifests itself, after field cooling from above the Néel temperature, by a shift $H_{\mathrm{EB}}$ of the hysteresis loop along the applied magnetic field axis and may be accompanied by an enhancement of the coercivity $H_{\mathrm{C}}[2,3]$. Since the FM-AFM interface is buried and the changes in the magnetization at interfaces are small, the description of its depth profile is very challenging.

Most of the investigation concerns the in-plane EB for which x-ray photoemission electron microscopy and x-ray magnetic linear or circular dichroism have revealed several interesting features about the spin behavior [4], like the correlation between a fraction of the pinned uncompensated interfacial spins with the exchange-bias field [5]. Polarized neutron reflectometry [6], as well as X-ray resonant magnetic scattering (XRMS) [7], also provided details about the in-plane magnetization depth profiles across the interfaces. In contrast, the studies of perpendicular EB are very scarce $[8,9]$ and only a few indirectly address the role of the magnetic organization at the interface [10,11]. Indeed, few techniques allow one to probe the perpendicular magnetization distribution in thin films. In specific cases, nuclear resonant scattering [12] can be sensitive to the out-of-plane magnetization distribution, and lateral resolution may be achieved with magnetic force microscopy [13], but none provide in-depth spatial resolution with chemical selectivity. It is therefore of interest to investigate the capability of XRMS to probe the in-depth out-of-plane magnetization in a perpendicular exchange coupled system and to examine whether the models developed for in-plane EB may apply to perpendicular EB. XRMS is known to be sensitive to the three directions of the magnetization with spatial, chemical, and orbital selectivity. However, up to date, XRMS in specular reflectivity geometry was only employed to investigate systems with in-plane magnetization [14].

In this Letter, we show that polarized soft-x-ray reflectivity allows the description of the out-of-plane magnetization distribution in the AFM-FM system $[\mathrm{NiO}(3 \mathrm{ML}) / \mathrm{CoO}(3 \mathrm{ML})]_{3} / \mathrm{Pt}-\mathrm{Co} / \mathrm{Pt}(111)$, exhibiting perpendicular EB. We observe that, above the blocking temperature, 1 monolayer (ML) of Co sandwiched between $\mathrm{Pt}$ layers induces magnetic order over $1 \mathrm{~nm}$ in the oxide layer. In the immediate interface the coupling is found ferromagnetic and antiferromagnetic above.

The sample was grown by alternate electron beam evaporation and studied in situ by surface x-ray diffraction (SXRD). One Co ML was deposited onto a clean Pt(111) substrate held at $543 \mathrm{~K}$. In such a case, Co atoms exchange with the $\mathrm{Pt}$ atoms to form a Pt-Co ferromagnetic layer with strong perpendicular magnetic anisotropy (PMA) [15]. Afterwards, $1 \mathrm{ML}$ Co was evaporated at $450 \mathrm{~K}$ and oxidized by exposure to $\mathrm{O}_{2}$. Repeating this procedure 3 times, an epitaxial $3 \mathrm{ML} \mathrm{CoO}(111)$ layer with in-plane bulk lattice constant was obtained. Following the same procedure, a $3 \mathrm{ML} \mathrm{NiO(111)} \mathrm{layer} \mathrm{was} \mathrm{grown} \mathrm{on} \mathrm{top} \mathrm{of} \mathrm{it.} \mathrm{This}$ $\mathrm{NiO} / \mathrm{CoO}$ sequence was repeated 3 times, yielding an AFM film that benefits from the high magnetocrystalline anisotropy constant of $\mathrm{CoO}$, with an intermediate Néel temperature $\left(T_{N}^{\mathrm{CoO}}=293 \mathrm{~K}<T_{N}<T_{N}^{\mathrm{NiO}}=523 \mathrm{~K}\right)[16]$.

Ex situ magnetic characterization of the AFM-FM system was carried out by polar magneto-optic Kerr effect (PMOKE) measurements (Fig. 1). The sample was field 

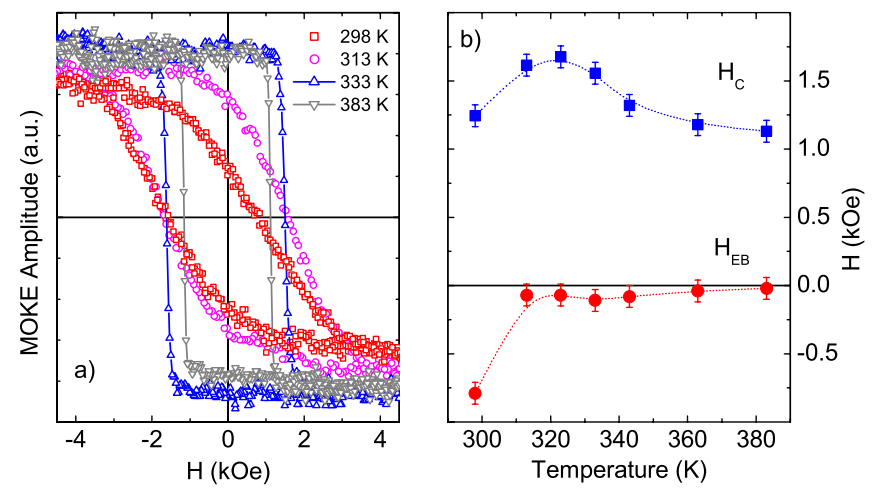

FIG. 1 (color online). (a) PMOKE hysteresis loops at different temperatures. (b) Temperature change of exchange bias and coercivity of the sample (the line is a guide for the eyes).

cooled from $383 \mathrm{~K}$ down to $298 \mathrm{~K}$ under $5 \mathrm{kOe}$. From $383 \mathrm{~K}$ to $323 \mathrm{~K}$, the hysteresis curves exhibit square loops with increasing $H_{\mathrm{C}}$ and essentially no $H_{\mathrm{EB}}$. From $323 \mathrm{~K}$ down to $298 \mathrm{~K}$, the loop becomes more and more elongated and shifted to the negative field value with decreasing $H_{\mathrm{C}}$.

The temperature below which the unidirectional anisotropy appears, defined as the blocking temperature, is inferred to be $T_{B} \approx 320 \mathrm{~K}$. Above $T_{B}$, the complete reversal of the out-of-plane magnetization of the Pt-Co ferromagnetic layer is observed. The increased coercivity $H_{\mathrm{C}}$, which

$$
f=-f_{c}\left(\begin{array}{cc}
1 & 0 \\
0 & \cos 2 \theta
\end{array}\right)+i f_{m}\left(\begin{array}{cc}
0 & m_{x} \cos \theta+m_{z} \sin \theta \\
-m_{x} \cos \theta+m_{z} \sin \theta & -m_{y} \sin 2 \theta
\end{array}\right) .
$$

The first term is the Thomson and resonant charge scattering with $f_{c}=f_{0}+f^{\prime}(E)+i f^{\prime \prime}(E)$ where $E$ is the photon energy. The second term is the resonant magnetic scattering where $f_{m}=m^{\prime}(E)+i m^{\prime \prime}(E)$ [20]. The sensitivity to the out-of-plane component of the magnetization unit vector $m_{z}$ occurs in the off-diagonal terms with a $\sin \theta$ dependence, where $\theta$ is the incident angle, indicating that sensitivity arises at high angular values.

The soft-x-ray specular reflectivity experiments were conducted on the SIM beam line at the Swiss Light Source using the RESOXS end station [21]. The sample was mounted on a cold finger and the magnetic saturation of the FM layer was achieved by a 4 kOe perpendicular permanent magnet brought to the sample surface. All measurements were carried out in remanence. The reflected intensity $I$ was recorded as a function of $\theta$ at different photon energies, and as function of $E$ at different $\theta-2 \theta$ settings. The dichroic difference $I^{+}-I^{-}$and the asymmetry ratio $R=\left(I^{+}-I^{-}\right) /\left(I^{+}+I^{-}\right)$were collected using 98\% left and right circularly polarized beam. Absorption spectra were collected simultaneously by recording the drain current as a function of $E$.

Figure 2 displays the energy dependence of the drain current, collected at an incidence of $90^{\circ}$, and of the specular reflectivity measured at $\theta=30^{\circ}$ and $42^{\circ}$ around the $\mathrm{Co}$ and $\mathrm{Ni} L_{3}$ edges. The spectral shape of the absorption is reaches a maximum of $1.7 \mathrm{kOe}$ around $T_{B}$, is related to long range AFM order appearing in the mixed oxide layer. It is worth noting that the extrapolated value of $H_{\mathrm{C}}$ for temperature above $T_{\mathrm{N}}, 1.2 \mathrm{kOe}$, equals the coercivity of this FM layer without the oxide [15]. Below $T_{B}$, the easy magnetization axis of the FM layer is no longer perpendicular. Upon field cooling, the AFM spins should align along the spin anisotropy axis that is closest to the applied magnetic field [9]. The $\mathrm{CoO}$ has a high magnetocrystalline anisotropy along the [117] axis and the Co spins are distributed on a cone surface, with half apex angle $43.3^{\circ}$, around the [111] growth direction. The orientation of the $\mathrm{NiO}$ magnetic moments is assumed to follow the $\mathrm{CoO}$ spins because of a lower magnetocrystalline anisotropy constant and a strong exchange interaction at the $\mathrm{CoO} / \mathrm{NiO}$ interface [17]. Hence, the change in the hysteresis loops is related to the reorientation of the Co spins in the FM layer due to exchange coupling with the oxide multilayer.

The x-ray resonant magnetic reflectivity exploits the magnetization sensitivity of the atomic scattering factor (ASF) at an absorption edge [18], which may be expressed on the basis of the two polarization states chosen either parallel or perpendicular to the scattering plane [19]:

essentially that of the $\mathrm{CoO}$ and $\mathrm{NiO}$ oxide, as expected from a total electron yield measurement [22]. The absorption spectra for pure Co and Ni metal are superimposed for comparison. In the reflectivity condition, the penetration depth is large enough to probe the buried Co layer, and the different spectral shapes illustrate the complex mixture of the real and imaginary components of the ASF [18].
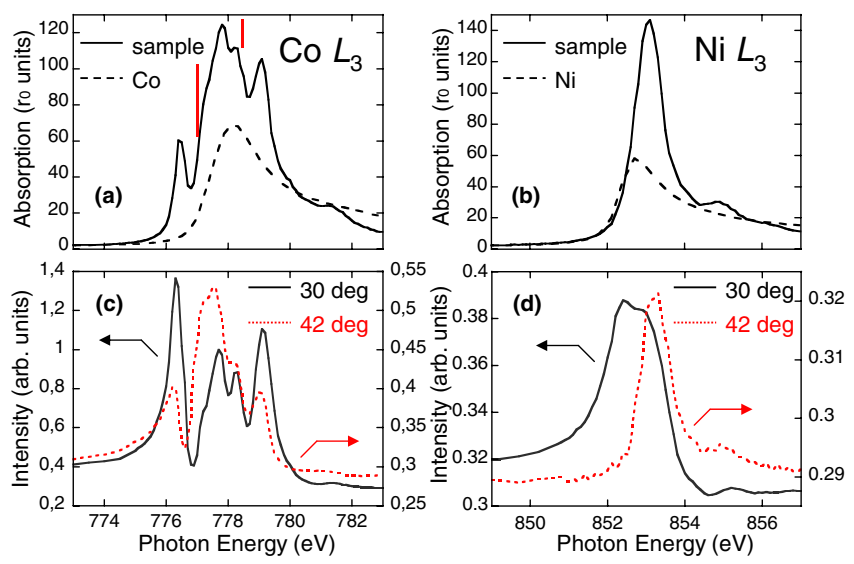

FIG. 2 (color online). (a),(b) Absorption spectrum (TEY) recorded at the $\mathrm{Co}$ and $\mathrm{Ni} L_{3}$ edge at $\theta=90^{\circ}$, and energy dependent intensity recorded at $\theta=30^{\circ}$ (c) and $42^{\circ}$ (d). 
We report here on measurements at $T=340 \mathrm{~K}$ right above $T_{B}$, where the FM layer has a strong PMA and where the interfacial magnetic coupling constrains the reversal process as indicated by the increased $H_{\mathrm{C}}$ (Fig. 1). Angle dependent reflected intensity (Fig. 3) was collected at $E=776.9$ and $778.5 \mathrm{eV}$ [see lines in Fig. 2(a)]. At both energies, a separation of the $I^{+}$and $I^{-}$curves is observed and is mainly due to the Co magnetization in the Pt-Co layer and potentially in the oxide layer. The asymmetry ratios are close to zero at small angles and exhibit a larger amplitude at high angles. This is in agreement with the geometrical dependence of the atomic scattering factor arising from an out-of-plane magnetization component. Numerical simulations displayed in Fig. 3 and discussed hereafter corroborate this interpretation.

Figures 4(a) and 4(c) show the differences $I^{+}-I^{-}$ between energy dependent intensities collected across the Co $L_{3}$ edge at $30^{\circ}$ and $42^{\circ}$ [the average charge intensity $\left(I^{+}+I^{-}\right) / 2$ is displayed in Fig. 2(c)]. The observation of a difference, exhibiting an opposite sign for each spectral feature under reversal of the applied magnetic field, proves the magnetic origin of the small dichroic signal. Such a difference is not a pure $\mathrm{x}$-ray magnetic circular dichroism (XMCD) signal but mixes $m^{\prime}, m^{\prime \prime}$ with different weighting factors depending on the geometrical conditions and on the charge ASF of the system [20]. Therefore, it is not straightforward to ascribe the spectral shape of the difference, which is more complex than expected for metallic Co atoms, to a net magnetization carried by the Co atoms in the $\mathrm{CoO}$ layers. However, a clear indication of the net magnetization in the AFM oxide layers comes from measurements at the Ni $L_{3}$ edge [Fig. 4(b) and 4(d)], which
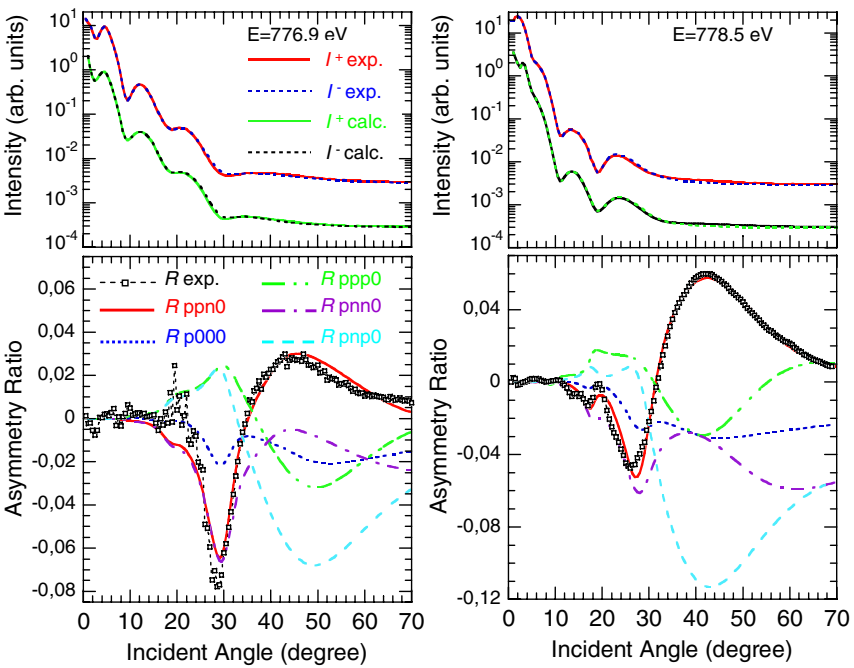

FIG. 3 (color online). Top panels: experimental and calculated specular reflected intensities with left and right circular light at 776.9 and $778.5 \mathrm{eV}$ with $H=0.4 \mathrm{~T}$ (the experimental data in the upper curves were multiplied by 10 for clarity). Bottom panels: experimental asymmetry ratios, best fit (Rppn0) and different models (see text). show a small net magnetic moment on $\mathrm{Ni}$ atoms. $\mathrm{Ni}$ atoms are magnetically active either due to an extended polarization through the first 3 ML Co layer or by contact with the PtCo interface due to discontinuity of this layer. Both situations indicate that the Co atoms in the oxide layer, at least close to the interface, are magnetically polarized.

In order to describe the spatial distribution of the Co magnetization in the oxide layer, magneto-optic calculations [23], extended in the soft-x-ray range, were carried out to reproduce the angular dependence of $I^{+}, I^{-}$, and $R$ (Fig. 3) [24]. The good agreement between experiments and calculations at two energies allows the description of the out-of-plane magnetization over a thickness larger than the ferromagnetic Pt-Co layer. Our model assumes that Co atoms in the oxide may be magnetically ordered at least up to the first $\mathrm{CoO} / \mathrm{NiO}$ interface, while the two topmost layers are not. The interference in $R$ at about $32^{\circ}$ indicates a magnetic thickness smaller than the total thickness of the oxide.

A first step concerns the determination of the structural parameters from the refinement of $\left(I^{+}+I^{-}\right) / 2$. The thicknesses (in nm), are $[\mathrm{NiO}(0.85 \pm 0.1) / \mathrm{CoO}(0.95 \pm 0.1)]_{2} /$ $\mathrm{NiO}(0.65 \pm 0.2) / \mathrm{CoO}(1.2 \pm 0.2) / \mathrm{Pt}-\mathrm{Co}(0.36 \pm 0.04) / \mathrm{Pt}$, where a periodic structure for the top four layers is kept in order to limit the number of parameters. The interfacial roughnesses are $0.02 \pm 0.01 \mathrm{~nm}$ for the $\mathrm{CoO} / \mathrm{PtCo}$ and in the range of $0.6 \pm 0.2 \mathrm{~nm}$ to $0.9 \pm 0.2 \mathrm{~nm}$ from the bottom to the top for the $\mathrm{Co}(\mathrm{Ni}) \mathrm{O} / \mathrm{Ni}(\mathrm{Co}) \mathrm{O}$ interfaces. These roughness parameters are in agreement with an island growth of the first Co oxide layers as inferred from SXRD and recent STM data.

The second step aims at the refinement of $R$, which requires one to model the magnetization profile. In order to take into account the change of the Co magnetic moment from $1.77 \mu_{B}$, in a thick film [24], to $2.07 \mu_{B}$ in the Pt-Co film [15], the magnetic resonant parameters are weighted

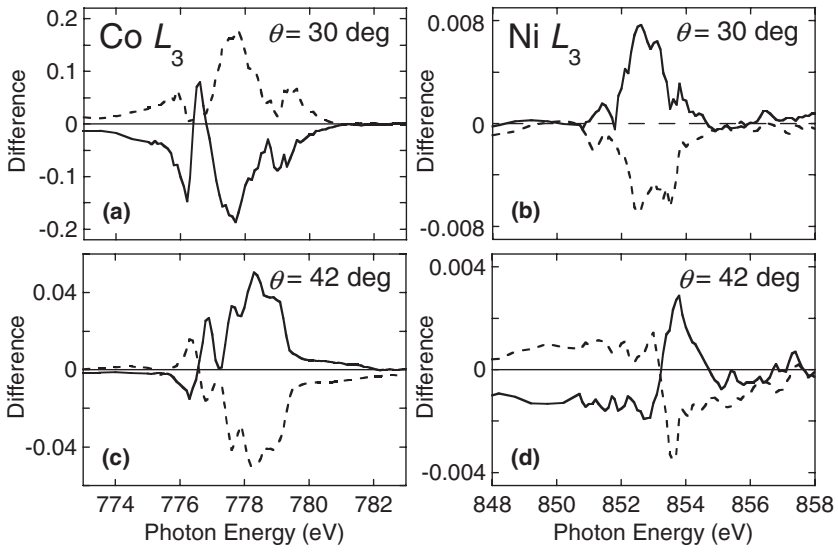

FIG. 4. Energy dependent differences obtained from reflectivity measurements with left and right circular light at $30^{\circ}$ (a),(b) and $42^{\circ}$ (c),(d) for Co and Ni $L_{3}$ edge with $H=+0.4 \mathrm{~T}$ (solids curves) and $H=-0.4 \mathrm{~T}$ (dashed curves). 
by a fixed factor 1.2 in the calculations. The refinement of the magnetic structure was performed by dividing the first $\mathrm{CoO}$ layer in three slices and by adjusting their thickness as well as the magnetic amplitudes carried by the oxidized Co atoms. The results at $E=776.9$ and $778.5 \mathrm{eV}$ indicate the out-of-plane magnetization is distributed over $1 \pm 0.1 \mathrm{~nm}$ in the oxide layer. It is coupled parallel to the metal Co in a $0.3 \pm 0.1 \mathrm{~nm}$ thick slice and then antiparallel in a $0.7 \pm$ $0.2 \mathrm{~nm}$ thick one with similar amplitude. The last slice $0.2 \pm 0.2 \mathrm{~nm}$ is found without net magnetization. The calculation of $R$ for other models yields large discrepancies (Fig. 3): only the Co metal is magnetized with a positive orientation, and there is no induced polarization for the three oxide slices $(p 000)$, the two first oxide slices are magnetized and coupled parallel ( $p p p 0)$, or antiparallel ( $p n n 0)$ to the FM layer, and the change of sign is opposite to the best model ( $p n p 0)$. This result can be understood as follows: the $\mathrm{Co}$ atoms in the first $\mathrm{CoO}$ slice on top of the PtCo layer, have a mixed electronic character in between metallic and oxidized state. Its net induced magnetization is likely to be dominated by the proximity of the Pt-Co layer and is ferromagnetically coupled to it. For the next $\mathrm{CoO}$ slice, the $\mathrm{Co}$ atoms are fully oxidized and the net magnetization is antiferromagnetically coupled to the FM layer as in [13]. A further step in this study would be to probe the extension of the Ni magnetization by the examination of the angular dependence of the reflected intensity at the Ni $L_{3}$ edge.

In conclusion, we demonstrate that the soft-X-ray resonant magnetic reflectivity measured over a wide angular range provides a direct way to probe out-of-plane magnetic profiles. It allows us to describe the extension and structure of the magnetic ordering induced, slightly above $T_{B}$, by an ultrathin Co FM layer, over a few oxide atomic layers in the AFM layer. This result for perpendicular EB is similar to those obtained for in-plane EB (induced magnetization in the AFM layer close to the interface) with, at difference, a depth profile exhibiting a change of sign related to the different coupling for the partially oxidized $\mathrm{Co}$ in the immediate interface and the fully oxidized Co above it. Future work is required to probe the magnetic profile changes at lower $T$ where EB develops and the magnetization of the FM layer rotates. The spin polarization of $\mathrm{Ni}$ and Co has already been observed in energy dependent measurements at $150 \mathrm{~K}$, indicating the presence of uncompensated spins at interface. However, at lower $T$, the magnetization at remanence is not saturated, which imposes one to perform the measurements under an applied magnetic field while keeping a total access to the scattering plane.

We thank Y. Souche, F. Nolting for valuable discussion, and S. Garaudée, as well as the beam line staff of X11MA for technical assistance. The work at SLS has been supported by the European Commission under the 6th
Framework Programme through the Key Action: Strengthening the European Research Area, Research Infrastructures, Contract No. RII3-CT-2004-506008.

*Present address: Institut für Allgemeine Physik, Technische Universität Wien, A-1040 Wien, Austria.

[1] J. B. Kortright et al., J. Magn. Magn. Mater. 207, 7 (1999).

[2] J. Nogues and I. K. Schuller, J. Magn. Magn. Mater. 192, 203 (1999).

[3] A.E. Berkowitz and K. Takano, J. Magn. Magn. Mater. 200, 552 (1999).

[4] H. Ohldag et al., Phys. Rev. Lett. 87, 247201 (2001).

[5] H. Ohldag et al., Phys. Rev. Lett. 91, 017203 (2003).

[6] M. R. Fitzsimmons et al., Phys. Rev. B 75, 214412 (2007).

[7] S. Roy et al., Phys. Rev. B 75, 014442 (2007).

[8] B. Kagerer et al., J. Magn. Magn. Mater. 217, 139 (2000).

[9] S. Maat et al., Phys. Rev. Lett. 87, 087202 (2001).

[10] S. M. Zhou et al., Phys. Rev. B 69, 024408 (2004).

[11] A. Baruth et al., Phys. Rev. B 74, 054419 (2006).

[12] R. Röhlsberger et al., Phys. Rev. B 67, 245412 (2003).

[13] P. Kappenberger et al., Phys. Rev. Lett. 91, 267202 (2003).

[14] C.-C. Kao et al., Phys. Rev. B 50, 9599 (1994); J. M. Tonnerre et al., J. Appl. Phys. 83, 6293 (1998); L. Sève et al., Phys. Rev. B 60, 9662 (1999); E. Dudzik et al., ibid. 62, 5779 (2000); M. Sacchi et al., ibid. 64, 012403 (2001); D. Haskel et al., Phys. Rev. Lett. 87, 207201 (2001); J. Geissler et al., Phys. Rev. B 65, 020405(R) (2001); Y. Choi et al., ibid. 70, 134420 (2004); M. Hecker et al., ibid. 72, 054437 (2005); S. Roy et al., Phys. Rev. Lett. 95, 047201 (2005).

[15] L. Giovanelli et al., J. Magn. Magn. Mater. 288, 236 (2005).

[16] J. A. Borchers et al., Phys. Rev. Lett. 70, 1878 (1993).

[17] M. J. Carey et al., J. Appl. Phys. 73, 6892 (1993).

[18] J. B. Kortright and Sang-Koog Kim, Phys. Rev. B 62, 12216 (2000).

[19] J.P. Hill and D. F. McMorrow, Acta Crystallogr. Sect. A 52, 236 (1996).

[20] J. M. Tonnerre et al., Phys. Rev. Lett. 75, 740 (1995).

[21] N. Jaouen et al., J. Synchrotron Radiat. 11, 353 (2004).

[22] T. J. Regan et al., Phys. Rev. B 64, 214422 (2001).

[23] J. Zak et al., Phys. Rev. B 43, 6423 (1991); 46, 5883(E) (1992).

[24] The $f^{\prime \prime}$ and $m^{\prime \prime}$ parameters of Co in the Pt-Co layer have been derived from the $\mathrm{x}$-ray-absorption spectroscopy (XAS) and XMCD spectra of a thick Co film, measured with a similar energy resolution. XAS data were scaled to the calculated atomic photoabsorption cross section far from the $L_{2,3}$ edges. For the Co in the oxide, the $f^{\prime \prime}$ parameter comes from the TEY measurements from the sample. No XMCD data were available to derive $m^{\prime \prime}$. We used the magnetic parameters of the metallic Co. At the chosen photon energies, the dichroic signal of the Co in the oxide is expected to have the same sign as the Co metal. Therefore, we do not comment on the amplitude of the magnetization in the $\mathrm{CoO}$ layer, but its sign. 